\title{
Nouvelles du Comité central
}

\section{Lors de sa séance d'octobre, le Comité central de la FMH a ...}

... approuvé le document de base du projet relatif à la «Révision de la statistique médicale»; le groupe de projet, placé sous la direction du département DDQ, y traite les questions relatives aux objectifs, aux questions et bases de la statistique de la FMH ainsi qu'à la structure du recensement et aux définitions;

... pris connaissance du fait que le canton de Neuchâtel s'est déclaré prêt à participer au projet-pilote ReMed; la Suisse alémanique est ainsi représentée par le canton de Thurgovie et la Suisse romande par le canton de Neuchâtel, pour le projet-pilote;

... décidé de contribuer financièrement au projet TMIplus, lequel vise, chez des patients du réseau médical urbain zmed, à pronostiquer les coûts de la santé au moyen d'un indicateur de morbidité modifié;

... pris bonne note des premières réflexions menées au sujet du «Blocage de l'accès à la pratique privée - Critères de qualité» (y compris le monitoring et la fourniture de prestations médicales) et décidé d'étendre à d'autres partenaires le projet relatif à la qualité;

... reçu des informations sur la première séance de la Commission des professions médicales (MEBEKO); l'une des questions importantes est de savoir si cette commission pourra, à l'avenir, fixer elle-même les conditions à remplir pour l'acquisition d'un titre par les détenteurs de diplômes étrangers non reconnus; pour la FMH, ces conditions doivent rester identiques aux programmes de formation postgraduée accrédités;

... décidé de se prononcer contre l'enregistrement obligatoire au registre du commerce des médecins installés et va entreprendre des activités de lobbying à ce sujet dans le cadre de l'Union suisse des professions libérales (USPL); les enregistrements au registre du commerce relèvent de la compétence du Conseil fédéral depuis que le droit des sociétés à responsabilité limitée (Sàrl) a été révisé;

... approuvé la prise de position de la FMH concernant le «Financement des institutions de prévoyance de droit public» adressée au DFI; la FMH soutient aussi bien l'adaptation juridique que l'adaptation financière proposées, à la condition que ces modifications ne chargent pas les employés;

... désigné Mme le Dr Raffaella VerzasconiZimmermann pour succéder à Mme le Dr Yvette Barbier à l'«Organe paritaire Convention Médecins-conseils» et à la «Commission de surveillance pour la formation postgraduée de médecin-conseil»;

... décidé de recommander à la CDS le Dr Florian Brunner, spécialiste en médecine physique et réadaptation à Zurich, pour la «Commission d'examens intercantonaux de chiropratique»;

... pris connaissance du fait que le domaine Tarifs a formé un groupe de projet Laboratoire, afin de suivre les développements de la QUALAB et d'élaborer une proposition sur la façon d'intégrer le laboratoire du praticien dans les «Directives sur le contrôle interne de la qualité» actuellement prévues;

... été mis au courant par O. Kappeler au sujet des «modèles basés sur des listes de médecins» proposés par des assureurs et de la discussion qui a eu lieu sur ce thème entre assureurs et corps médical à fin septembre; un article paru dans le $\mathrm{n}^{\circ} 44 \mathrm{du}$ BMS donne une vue d'ensemble détaillée de la situation;

... accepté la cotisation annuelle que la FMH verse à RADIX Promotion de la santé à titre de membre du Comité de patronage de cette fondation. 